re-analyse our data in collaboration with Dr Evans and his group (including the alprazolam data) to see whether factor analysis can give us new insight and thereby decrease the fear of illusion. We also hope that the results can be published in this journal.

Hamitton, M. (1977) Standard criteria for clinical assessment in psychopharmacology. In Evaluation of New Drugs in Clinical Psychopharmacology (eds A. Bertelli, G. B. Cassano, P. Castrogiovanni et al), pp. 101-105. Barcelona: J. R. Prous.

Department of Psychiatry

P. BECH

Frederiksborg General Hospital

DK-3400 Hillerod

Denmark

Danish Institute of Educational Research

P. Allerup

Department of Psychiatry

W. MAIER

University of Mainz

Germany

Psychiatric University Clinic

Munich

Germany

Massachusetts General Hospital

Boston

USA

University of Madrid

Spain

M. Albus

P. LAVORI

J. L. Ayuso

\section{The meaning of insight}

SIR: Drs Markova \& Berrios provide an adequate historical account of the search for a definition of insight (Journal, June 1992, 160, 850-860). However, it seems to us that their attempt to be all-inclusive means that their conclusions offer little guidance to the clinical psychiatrist. 'Insight' in clinical psychiatry will not mean the same as in philosophy, and need not mean the same as the psychodynamic use of the term to indicate a deep level of selfknowledge. The authors appear to recognise this, and state that "whether insight itself is adequately defined may not be as important as reliably measuring perhaps only aspects of the concept". Furthermore, they feel that "it would seem appropriate to grade the level of 'insight' ".

In the context of a diagnosed psychiatric disorder, we propose a pragmatic and hierarchical definition of insight whose predictive validity is amenable to testing:
Level 1: the patient is aware of change in perceptual experiences, cognitive processes, emotions, or behaviours

Level 2: the patient has a feeling of disease engendered by these changes

Level 3: the patient gives verbal recognition that the changes causing disease are pathological, i.e. they amount to an illness

Level 4: the patient acts on this in a manner appropriate to his/her intellectual and cultural background by seeking treatment, or complying with treatment, from a psychiatrist.

This schema would seem to us to offer a practical and testable alternative to previous attempts at a clinically useful definition of insight (e.g. McEvoy et al, 1989; David, 1990). It accommodates, for example, the patient with a psychotic disorder who develops a delusional system to account for perceived unpleasant changes, and whose insight is therefore assessed at level 2, and the patient with a neurotic disorder who self-deceives by denying the psychological nature of the illness, and whose insight is therefore assessed at level 3.

By qualifying the definition "in the context of a diagnosis of psychiatric disorder" we exclude the purely neurological conditions described by Drs Markova \& Berrios. It is possible that the study of these conditions will ultimately cast light on the mechanisms of insight, but they have little practical relevance in routine clinical psychiatry.

DAvid, A. S. (1990) Insight and psychosis. British Journal of Psychiatry, 156, 798-808.

McEvoy, J. P., Apperson, L. J., Appelbaum, P. S., et al (1989) Insight in schizophrenia. Its relationship to acute psychopathology. Journal of Nervous and Mental Disease, 177, $42-47$.

Department of Psychiatry

Peter Raven

RichaRd MULLEN

Claire Capstick

Institute of Psychiatry

De Crespigny Park

Denmark Hill

London SE5 8AF

\section{Benzodiazepines with ECT}

SIR: In the article by Cohen \& Lawton (Journal, April $1992,160,545-546)$ they mentioned that they gave electroconvulsive therapy (ECT) using an Ectron duopulse constant-current machine at waveform 2 with a stimulus lasting for 1.5 seconds. In the table about stimulus delivered by machines by Russell (1988), a duopulse machine giving waveform 2 for 
1.5 seconds is not a constant-current machine, and in a recent article by Pippard (Journal, May 1992, 160, 621-637) a stimulus length of 1.5 seconds does not appear to be available from constant-current ECT machinery. I wonder if the stimulus dosage given is actually known at all. Could more data about the stimulus dosage given at ECT offer an explanation? The complex relationship between stimulus dosing and seizure duration may offer an explanation. The first course of ECT in their patient may have produced no therapeutic effect despite producing convulsions because the dosage did not exceed the seizure threshold adequately for therapeutic effect. There could with time, or the waning benzodiazepine effect, have been a change in seizure threshold in their patient. I think it is about time that ECT was not regarded as a black hole and that the dosage received by patients was adequately charted for scientific study of its effect.

RusselL, R. J. (1988) Stimuli used in ECT. In Current Approaches to ECT (eds J. C. Malkin \& S. Brandon), pp. 58-60. Southampton: Duphar Medical Relations.

Wonford House Hospital

R. F. HILL

Dryden Road

Exeter EX2 5AF

AUTHORs' REPLY: Dr Hill is quite right that the dosage of ECT received by patients should be known and recorded, and I am afraid that we cannot be as precise as he would like. However, the essential point we make is that the same technique with the same machine was used on each occasion, and that the presence of benzodiazepines appeared to have prevented the response to ECT. We are not sufficiently expert to comment on the exact reason for this, but the lesson is that these drugs must be discontinued some time before giving ECT to depressed patients.

Samuel. I. Cohen

The Royal London Hospital Trust Claire lawton

St Clement's

$2 A$ Bow Road

London E3 4LL

\section{ECT for young people}

SIR: There has been recent controversy about the use of electroconvulsive therapy (ECT) to treat young people. Consequently, we reviewed the frequency of, and the indications for, the use of ECT in patients under 18 years of age treated at the Royal Edinburgh Hospital over the past ten years. The hospital is a psychiatric teaching hospital that serves a population of $\mathbf{4 4 0 0 0 0}$ and is the only site in Edinburgh in which ECT could be given to young people. Written ECT records are still available from January 1982 and follow-up of young people treated by ECT was possible by case-note review up to June 1992

Five people under 18 years were treated with ECT. All were female and all were 17 years old at the time. Patient 1 was treated in 1982 for a delusional depressive illness that had not responded to a tricyclic antidepressant alone or in combination with a neuroleptic drug. Subsequently, the patient had 17 admissions to the hospital and the diagnosis was revised to one of schizophrenia. Patient 2 was treated in 1986 for the depressive phase of a manicdepressive illness that had not responded to a tricyclic antidepressant alone or in combination with lithium carbonate. Patient 3 was treated in 1987 for depressed mood associated with delusions, but further details were unavailable because of the loss of the relevant casenotes. The patient remained in contact with the psychiatric services and the diagnosis was revised to one of schizophrenia. Patient 4 was treated in 1990 for a post-partum delusional depressive illness that had not responded to two different tricyclic antidepressants. Patient 5 was treated in 1991 for a delusional depressive illness that had not responded to two different tricyclic antidepressants. The diagnosis was revised to one of schizophrenia during follow-up.

The average frequency of the use of ECT in people under 18 years in Edinburgh was one case every two years. The indications were always depression associated either with psychotic features or manic-depressive illness.

The Royal College of Psychiatrists has not published guidelines for the use of ECT in young people, although the ECT Sub-Committee is presently revising the guidelines on the practical administration of ECT. The American Psychiatric Association Task Force on ECT (1990) recommended that ECT should never be used as a primary treatment for children or adolescents. ECT was recommended where the index illness had not responded to treatment, when there was a need for a rapid and definitive improvement in the illness for either medical or psychiatric reasons, or where ECT was likely to produce less severe adverse effects. There was no evidence that ECT was used inappropriately in the present review based on these APA recommendations. The APA also recommends that an independent psychiatrist experienced in treating adolescents reviews the indication for ECT, and this would be one area of practice that falls short of the recommendations. 